\author{
Ecología
}

\title{
Estructura de la comunidad ictioplanctónica en la laguna de Tamiahua, Veracruz, México
}

\author{
Structure of the ichthyoplankton community in Tamiahua Lagoon, Veracruz, Mexico \\ Alberto Ocaña-Luna* y Marina Sánchez-Ramírez \\ Laboratorio de Ecología, Departamento de Zoología, Escuela Nacional de Ciencias Biológicas, Instituto Politécnico Nacional, México D. F., México
}

Recibido el 18 de mayo de 2015; aceptado el 25 de septiembre de 2015

Disponible en Internet el 28 de febrero de 2016

\begin{abstract}
Resumen
La laguna de Tamiahua es la tercera laguna más grande de México. En la última década sus 2 bocas han sido dragadas y sus escolleras rehabilitadas para mantener la comunicación permanente con el mar, lo que permite el libre flujo de especies. Se realizaron muestreos de zooplancton en 22 estaciones durante los meses de febrero, mayo, agosto y noviembre de 2011, con una red estándar de $500 \mu \mathrm{m}$; simultáneamente se midió la salinidad y la temperatura superficial del agua. Se determinaron 40 especies pertenecientes a 37 géneros y 19 familias de peces. Lutjanus campechanus, Chasmodes bosquianus, Ctenogobius shufeldti, Microgobius thalassinus y Citharichthys arctifrons son nuevos registros para la laguna. Las especies dominantes fueron Anchoa mitchilli, Lagodon rhomboides, Membras martinica, Lupinoblennius nicholsi, Ctenogobius boleosoma y Brevoortia gunteri. En febrero la riqueza específica fue mayor (26) por el ingreso de especies marinas, mientras que la diversidad fue mínima (2.13) por la gran abundancia de L. rhomboides, A. mitchilli y B. gunteri del grupo iv en el dendrograma de asociación de larvas. La composición larvaria de la laguna de Tamiahua presentó una similitud del $61 \%$ al compararla con años anteriores, antes del dragado de la boca de Tampachichi. Derechos Reservados $\odot 2015$ Universidad Nacional Autónoma de México, Instituto de Biología. Este es un artículo de acceso abierto distribuido bajo los términos de la Licencia Creative Commons CC BY-NC-ND 4.0.
\end{abstract}

Palabras clave: Larvas de peces; Huevos; Laguna costera; Diversidad; Asociaciones; Golfo de México

\section{Abstract}

Tamiahua Lagoon is the third largest lagoon in Mexico. In the last decade their 2 inlets have been dredged and their jetties re-built to maintain constant communication with the sea, allowing the free flow of species. Zooplankton samplings were performed in 22 stations during the months of February, May, August and November of 2011, with a standard net of 500 microns. Simultaneously, salinity and surface temperature were measured. Forty species belonging to 37 genera and 19 families of fish were determined. Lutjanus campechanus, Chasmodes bosquianus, Ctenogobius shufeldti, Microgobius thalassinus and Citharichthys arctifrons are new records for this lagoon. The dominant species were Anchoa mitchilli, Lagodon rhomboides, Membras martinica, Lupinoblennius nicholsi, Ctenogobius boleosoma and Brevoortia gunteri. In February the species richness (26) was the highest one due to the entry of marine species, while diversity was low (2.13) due to the abundance of $L$. rhomboides, A. mitchilli and B. gunteri, of the group IV in the dendrogram of association of larvae. The larval composition from the Tamiahua lagoon presented a similarity of $61 \%$ when compared to previous years, before the dredging of the Tampachichi inlet.

All Rights Reserved @ 2015 Universidad Nacional Autónoma de México, Instituto de Biología. This is an open access item distributed under the Creative Commons CC License BY-NC-ND 4.0.

Keywords: Fish larvae; Eggs; Coastal lagoon; Diversity; Assemblages; Gulf of Mexico

\section{Introducción}

\footnotetext{
* Autor para correspondencia.

Correo electrónico: ja_ocanaluna@ hotmail.com (A. Ocaña-Luna).

La revisión por pares es responsabilidad de la Universidad Nacional Autónoma de México.
}

Las lagunas costeras son cuerpos de agua someros, semicerrados, con conexión al mar por algún tipo de barrera, que presentan un continuo intercambio de materiales y organismos (AyalaCastañares y Phleger, 1969; Lankford, 1977); en ellas, gran 
cantidad de peces, entre otros organismos, utilizan estos sistemas como zonas de crecimiento, alimentación, refugio, reproducción o para completar su ciclo de vida de especies migratorias marinas y de aguas continentales (Ayala-Castañares y Phleger, 1969; Day y Yáñez-Arancibia, 1982).

Las bocas de estos sistemas son de gran importancia, ya que permiten el intercambio de agua marina y el ingreso de especies de interés comercial, donde las alteraciones en el volumen del flujo de agua dulce es uno de los principales factores de estrés (Sklar y Browder, 1998), así como su naturaleza intermitente (Kjerfve, 1994; Kjerfve y Magill, 1989), que influyen en la riqueza, la diversidad y la dominancia de peces: estuarinorresidentes y marinos estuarinodependientes (Strydom, Whitfield y Wooldridge, 2003). Al respecto se ha observado que la apertura permanente de las bocas, como en la laguna La Machona, Tabasco, donde la boca de Panteones de origen artificial fue abierta en 1975 (Gómez-Aguirre y Reséndez-Medina, 1986), provoca un aumento en el número de especies de peces estenohalinas de origen marino y una disminución de las estuarinas temporales o permanentes (Salvadores-Baledón y ReséndezMedina, 1990).

En la laguna de Tamiahua, en la década del 2000 se iniciaron los trabajos de rehabilitación y prolongación de escolleras de las bocas Corazones y Tampachichi debido al frecuente azolve por los sedimentos provenientes del río Pánuco (SagarpaConapesca, 2007, 2008). En esta laguna convergen 2 provincias oceánicas, la carolineana al norte y la caribeña al sur; la primera presenta especies de clima templado-cálido y la segunda está expuesta a corrientes de agua cálida que es llevada hacia el norte por el sistema de corrientes del golfo y se caracteriza por la presencia de especies tropicales (Briggs, 1995). En ella, AbarcaArenas, Franco-López, González-Gándara y Silva-López (2012) registran 143 especies de peces adultos, mientras que en etapas larvarias Sanvicente-Añorve, Sánchez-Ramírez, Ocaña-Luna, Flores-Coto y Ordóñez-López (2011) mencionan 32.

En la región norte del estado de Veracruz se han desarrollado algunas investigaciones sobre ictioplancton en lagunas costeras: en la laguna de Pueblo Viejo, Sánchez-Ramírez y Ocaña-Luna (2002) estimaron la biomasa en desove de Anchoa mitchilli; en la laguna de Tampamachoco, Ocaña-Luna y Sánchez-Ramírez $(1998$, 2003) analizaron la alimentación de larvas de esciénidos y la diversidad de larvas de peces, respectivamente; en la laguna de Tamiahua, Flores-Coto, Barba-Torres y Sánchez-Robles (1983) dan a conocer la estructura de la comunidad ictioplanctónica, mientras que Gaspar-Dillanes, Sánchez-Iturbe, González-Yoval y López-García (1995) y Díaz-Ávalos, Barba-Torres y GasparDillanes (2003) evaluaron la biomasa en desove y los factores ambientales que influyen en la abundancia de A. mitchilli; por otro lado, Sanvicente-Añorve et al. (2011) realizaron un estudio sobre la metacomunidad de larvas de peces en lagunas costeras del golfo de México y el Caribe mexicano. Debido a las modificaciones del ambiente y a los pocos trabajos realizados en el sistema, en la presente investigación se analizan la abundancia estacional y la estructura de la comunidad ictioplanctónica a través de un ciclo anual (2011) en la laguna de Tamiahua, posterior al dragado de canales, la rehabilitación y la prolongación de escolleras de la boca de Tampachichi.

\section{Materiales y métodos}

La laguna de Tamiahua se localiza en el litoral occidental del golfo de México, entre los $21^{\circ} 06^{\prime}$ y $22^{\circ} 05^{\prime} \mathrm{N}$ y los $97^{\circ} 22^{\prime}$ y $97^{\circ} 46^{\prime} \mathrm{O}$, con una orientación NNO a SSE, paralela a la línea de costa, entre los ríos Pánuco al norte y Tuxpan al sur, con los que se comunica por sus canales Chijol y Tampamachoco, respectivamente. La laguna tiene una superficie aproximada de 88,000 ha, con $85 \mathrm{~km}$ de longitud y $18 \mathrm{~km}$ de anchura máxima, y por su extensión es la tercera laguna más grande de México (Contreras-Espinosa, 1993). La laguna está separada del golfo de México por una barrera arenosa de forma angular denominada Cabo Rojo, cuya longitud máxima es de $130 \mathrm{~km}$, su anchura máxima de $6 \mathrm{~km}$ y la mínima de $500 \mathrm{~m}$, que se originó a partir de un arrecife sepultado bajo ella y que podría ser sucesora de una barrera antigua representada actualmente por parte de las islas Juan A. Ramírez y del Toro; los sedimentos predominantes son limos y arcillas, excepto para el sotavento de la barrera, en donde existen arenas; a la laguna desembocan varios ríos, la mayoría de flujo estacional formando esteros, entre los que sobresalen La Laja, Cucharas, Tancochín y Tampache (AyalaCastañares, Cruz, García-Cubas, Jr. y Segura, 1969). Presenta 2 bocas, al norte la boca de Tampachichi, abierta de forma artificial en 1978, que requería ser dragada regularmente para mantenerla abierta, y al sur la boca de Corazones, de origen natural y permanentemente abierta (Rodríguez-de la Cruz y Palacio-Fest, 1996); ambas bocas han sido dragadas y sus escolleras prolongadas y rehabilitadas (Sagarpa-Conapesca, 2007, 2008).

En la laguna de Tamiahua se realizaron muestreos de zooplancton durante los meses de febrero, mayo, agosto y noviembre de 2011, en 22 estaciones distribuidas de la siguiente forma: 10 en la región norte desde Tampico Alto hasta el canal de Burros, al sur de la isla Juan A. Ramírez, y 12 en la región sur desde la desembocadura del estero Cucharas e isla del Toro hasta la boca de Corazones (fig. 1). Los arrastres fueron superficiales de 5 min de duración, con una red estándar de zooplancton de luz de malla de $500 \mu \mathrm{m}$, longitud de manga de $2 \mathrm{~m}$ y diámetro de la boca de $0.5 \mathrm{~m}$, a la cual se le adaptó un flujómetro General Oceanics para cuantificar el volumen de agua filtrado; las muestras de zooplancton se fijaron en formalina al $4 \%$, neutralizada con borato de sodio. Simultáneamente se registró la salinidad y la temperatura superficial del agua $\left({ }^{\circ} \mathrm{C}\right)$ y las estaciones se georreferenciaron con un GPS.

Se separó el total de huevos y larvas de peces de las muestras de zooplancton; los huevos fueron determinados a nivel de orden y familia, en el caso de las familias Engraulidae y Atherinopsidae a especie, mientras que la mayoría de las larvas lo fueron a nivel de especie, con excepción de las larvas vitelinas del orden Perciformes. Los ejemplares se cuantificaron y la densidad se expresó como el número de organismos $/ 100 \mathrm{~m}^{3}$. Posteriormente, se analizaron algunos parámetros ecológicos, como: riqueza, diversidad de Shannon-Wiener (Shannon y Weaver, 1963) utilizando $\log _{2}$, dominancia (Simpson, 1949), equidad (Pielou, 1975) -para estos análisis se excluyeron las larvas de: Microgobius spp., Clupeiformes, vitelinas de Perciformes e indeterminadas $-\mathrm{y}$ para determinar las especies dominantes se 


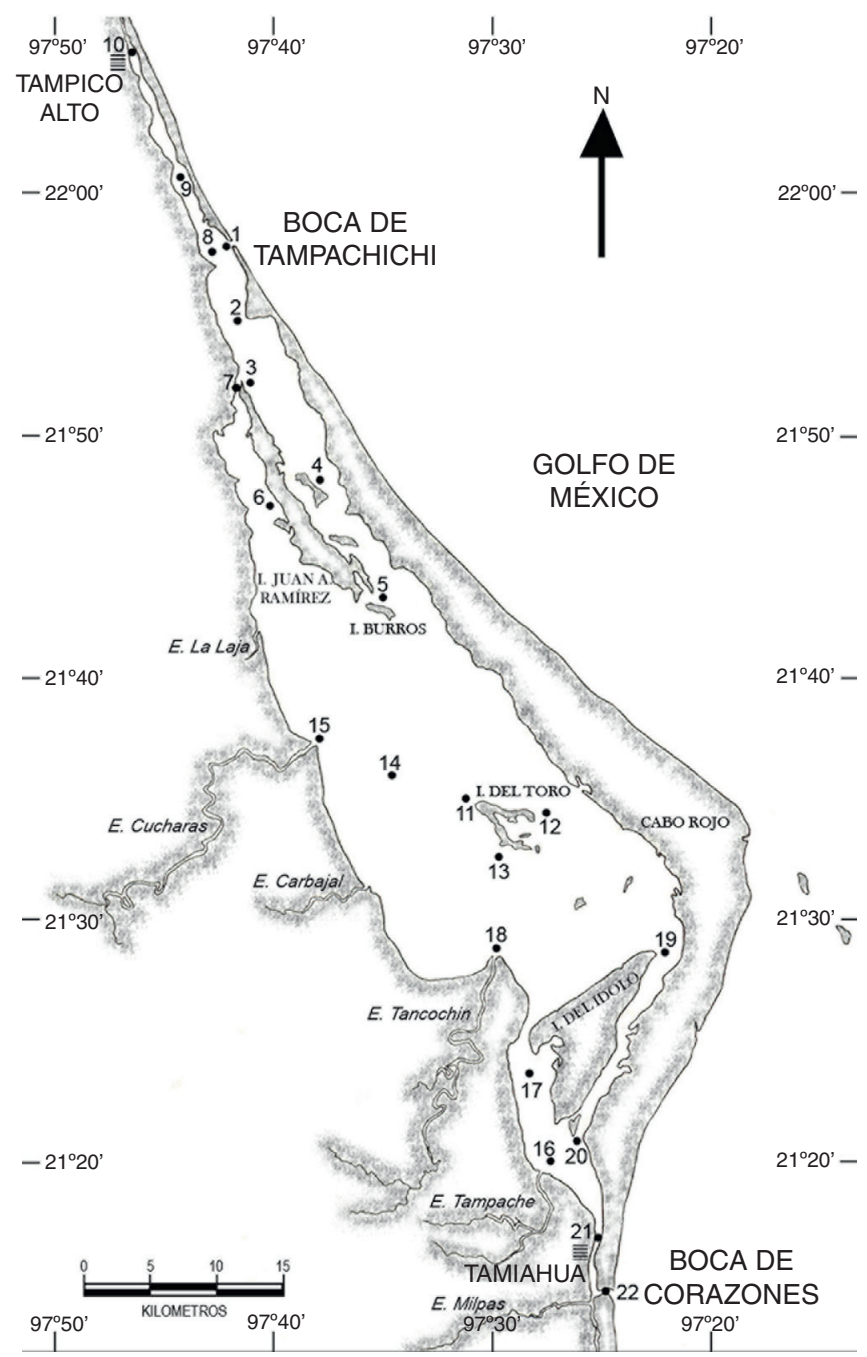

Figura 1. Área de estudio y ubicación de estaciones de muestreo en la laguna de Tamiahua, Veracruz, México (2011).

utilizó el índice de valor de importancia (IVI) (Krebs, 1985). Por otro lado, se relacionó la densidad de las especies dominantes con la temperatura y la salinidad del agua a través de la correlación de rangos de Spearman (Zar, 2010).

Para comparar la fauna ictioplanctónica de las subregiones norte y sur de la laguna de Tamiahua se utilizó el índice de similitud de Schoener (Schoener, 1970), que considera la proporción de la densidad de las especies en la captura total, como ha sido señalado por Paperno, Mille y Kadison (2001). Por otro lado, se calculó el coeficiente de similitud de Sorensen (Sorensen, 1948) para comparar la riqueza de especies de larvas de peces de la laguna de Tamiahua con la laguna Madre, Tamaulipas (Sánchez-Ramírez y Ocaña-Luna, 2015); laguna de Tamiahua (Sanvicente-Añorve et al., 2011) y laguna de Tampamachoco, Veracruz (Ocaña-Luna y Sánchez-Ramírez, 2003). Finalmente, se analizó la conformación de grupos en un dendrograma de asociación de especies utilizando el índice de Bray-Curtis (Bray y Curtis, 1957) a partir de los datos de densidad transformados $(\ln [\mathrm{x}+1])$, para lo que se utilizó el programa Past versión 2.01 (Hammer, Harper y Ryan, 2001) con el método de grupos pareados.
Tabla 1

Variación promedio \pm desviación estándar de la salinidad y temperatura superficiales registradas en la laguna de Tamiahua, Veracruz, México (2011).

\begin{tabular}{lllll}
\hline Mes/Parámetro & \multicolumn{3}{l}{ Temperatura $\left({ }^{\circ} \mathrm{C}\right)$} & \\
\cline { 2 - 4 } & Mínima & Máxima & Promedio & DE \\
\hline Febrero & 13.0 & 18.5 & 15.0 & 1.9 \\
Mayo & 25.0 & 29.5 & 27.8 & 1.1 \\
Agosto & 24.0 & 34.5 & 31.9 & 2.2 \\
Noviembre & 24.0 & 27.0 & 25.5 & 0.9 \\
& & & & \\
& Salinidad (ups) & & & DE \\
\cline { 2 - 4 } & Mínima & Máxima & Promedio & \\
\hline Febrero & 15.0 & 35.0 & 22.3 & 6.7 \\
Mayo & 21.0 & 37.0 & 31.0 & 5.2 \\
Agosto & 2.0 & 37.0 & 25.1 & 8.4 \\
Noviembre & 17.0 & 36.0 & 28.6 & 4.2 \\
\hline DE: desviacion & & & &
\end{tabular}

DE: desviación estándar.

\section{Resultados}

La temperatura promedio mensual del agua superficial fluctuó entre $15.0^{\circ} \mathrm{C}$ en febrero y $31.9^{\circ} \mathrm{C}$ en agosto; las temperaturas más bajas se registraron durante febrero. La salinidad promedio mensual osciló entre 22.3 ups en febrero y 31.0 ups en mayo (tabla 1).

\section{Riqueza específica}

Se recolectaron un total de 28,644 huevos y 1,883 larvas de peces, de los cuales se determinaron 19 familias, 37 géneros y 40 especies. Las familias con mayor número de especies fueron Gobiidae y Sciaenidae con 7 y 6, respectivamente. Se consideran nuevos registros para la laguna de Tamiahua a Lutjanus campechanus, Chasmodes bosquianus, Ctenogobius shufeldti, Microgobius thalassinus y Citharichthys arctifrons. En febrero y mayo se presentó la mayor riqueza (tabla 2).

\section{Abundancia de huevos}

Durante los meses de mayo y agosto se observó la mayor densidad de huevos, con el $88.07 \%$ del total anual. La mayor abundancia relativa correspondió a A. mitchilli (45.31\%), Perciformes (44.48\%) y Cetengraulis edentulus $(7.57 \%)$, que en conjunto representan el $97.36 \%$. Anchoa mitchilli presentó un amplio periodo de desove en la laguna, principalmente en agosto $\left(839.71 \mathrm{org} / 100 \mathrm{~m}^{3}\right)$, cuando se presentan mayores temperaturas (tablas 1 y 2), mientras que $C$. edentulus desova en el mar y sus huevos penetran con la masa de agua marina; estos se distribuyen desde la boca de Tampachichi hasta el sur de la isla Juan A. Ramírez de mayo a noviembre, con la mayor abundancia en mayo (146.08 huevos $/ 100 \mathrm{~m}^{3}$ ).

\section{Abundancia de larvas}

La mayor densidad de larvas se presentó en febrero, con un $53.92 \%$, y la mínima en noviembre, con un $6.70 \%$ del total anual. 
Tabla 2

Abundancia promedio mensual (número de organismos $/ 100 \mathrm{~m}^{3}$ ) y abundancia relativa (\%) de huevos y larvas de peces. Laguna de Tamiahua, Veracruz, México (2011).

\begin{tabular}{|c|c|c|c|c|c|}
\hline \multicolumn{6}{|c|}{ Huevos } \\
\hline Elopidae & & 0.09 & & & 0.004 \\
\hline Anchoa hepsetus & 0.04 & 0.15 & & 0.29 & 0.019 \\
\hline Anchoa mitchilli & 0.17 & 128.10 & 839.71 & 167.45 & 45.310 \\
\hline Cetengraulis edentulus & & 146.08 & 30.43 & 13.12 & 7.567 \\
\hline Perciformes & 53.87 & 854.87 & 149.75 & 56.18 & 44.481 \\
\hline Achiridae & & 44.89 & 12.14 & 7.59 & 2.578 \\
\hline
\end{tabular}

\begin{tabular}{|c|c|c|c|c|c|c|}
\hline Taxa & Febrero & Mayo & Agosto & Noviembre & $\%$ & IVI \\
\hline Elops saurus & 0.05 & & & & 0.04 & 1.19 \\
\hline Anchoa hepsetus & 0.08 & 0.28 & & & 0.29 & 2.59 \\
\hline Anchoa mitchilli & 17.60 & 7.05 & 13.77 & 2.84 & 33.57 & 38.16 \\
\hline Brevoortia gunteri & 7.78 & & & & 6.33 & 7.48 \\
\hline Opisthonema oglinum & & 0.20 & & & 0.16 & 1.31 \\
\hline Membras martinica & 1.54 & 0.94 & 1.38 & 0.79 & 3.78 & 8.38 \\
\hline Microphis brachyurus lineatus & & & 0.06 & & 0.05 & 1.20 \\
\hline Syngnathus louisianae & 1.09 & 0.20 & & & 1.05 & 3.35 \\
\hline Syngnathus scovelli & 0.83 & 0.21 & 0.15 & 0.32 & 1.23 & 5.83 \\
\hline Serranidae & & 0.07 & & & 0.06 & 1.21 \\
\hline Oligoplites saurus & & 0.36 & 0.27 & & 0.51 & 2.81 \\
\hline Trachinotus falcatus & & 0.06 & & & 0.05 & 1.20 \\
\hline Diplodus sp. & 0.05 & & & & 0.04 & 1.19 \\
\hline Lagodon rhomboides & 37.94 & 2.72 & 0.83 & & 33.75 & 37.20 \\
\hline Bairdiella chrysoura & 0.17 & 0.39 & 0.33 & 0.10 & 0.81 & 5.40 \\
\hline Cynoscion arenarius & & 0.38 & 0.07 & 0.71 & 0.94 & 4.39 \\
\hline Cynoscion nebulosus & & 0.73 & 0.42 & 0.25 & 1.14 & 4.59 \\
\hline Leiostomus xanthurus & 1.15 & & & & 0.94 & 2.08 \\
\hline Micropogonias undulatus & 0.05 & & & 0.17 & 0.18 & 2.48 \\
\hline Stellifer lanceolatus & & & & 0.06 & 0.05 & 1.20 \\
\hline Chasmodes bosquianus ${ }^{\mathrm{a}}$ & 0.30 & 0.20 & 1.14 & 0.06 & 1.38 & 5.98 \\
\hline Lupinoblennius nicholsi & 0.05 & 1.21 & 2.50 & 0.48 & 3.45 & 8.05 \\
\hline Gobiesox strumosus & 0.06 & & & & 0.05 & 1.20 \\
\hline Dormitator maculatus & 0.05 & 0.21 & 0.12 & & 0.31 & 3.76 \\
\hline Eleotris pisonis & 0.19 & & & & 0.15 & 1.30 \\
\hline Citharichthys arctifrons ${ }^{\mathrm{a}}$ & 0.05 & & & & 0.04 & 1.19 \\
\hline Achirus lineatus & & 0.44 & 0.39 & 0.77 & 1.30 & 4.75 \\
\hline Trinectes maculatus & & & & 0.31 & 0.25 & 1.40 \\
\hline Clupeiformes & & 0.09 & & & & \\
\hline Perciformes vitelinas & & 8.43 & 0.53 & 0.24 & & \\
\hline Indeterminadas & & & 0.08 & & & \\
\hline
\end{tabular}


Tabla 2 (continuación)

\begin{tabular}{lccccc}
\hline & \multicolumn{3}{c}{ Larvas } \\
\hline Taxa & Febrero & Mayo & Agosto & Noviembre & $\%$ \\
\hline Riqueza de especies & 26 & 25 & 19 & 17 & 3.21 \\
Diversidad & 2.13 & 3.30 & 2.32 & 0.17 & \\
Dominancia & 0.35 & 0.18 & 0.38 & 0.78 \\
Equidad & 0.45 & 0.71 & 0.55 & \\
\hline
\end{tabular}

IVI: índice de valor de importancia.

a Nuevos registros para la laguna de Tamiahua.

Las especies con mayor abundancia fueron Lagodon rhomboides (33.75\%), A. mitchilli (33.57\%) y Brevoortia gunteri (6.33\%) (tabla 2), que en conjunto representaron el $73.65 \%$ de la abundancia total anual. De las especies restantes, 21 fueron exclusivas de alguna época con abundancias muy bajas, de entre el 0.04 y el $0.94 \%$, y en conjunto constituyeron el $3.37 \%$. La mayoría de las especies fueron recolectadas en condiciones mesopolihalinas, con excepción de A. mitchilli, que se recolectó en salinidades de 2 a 37 ups (oligoeuhalinas), predominando en condiciones mesoeuhalinas; A. mitchilli, Dormitator maculatus y Gobiosoma bosc se registraron en agosto en la salinidad más baja ( 2 ups), al norte del sistema frente a Tampico Alto, en baja densidad.

\section{Relación entre densidad de huevos y/o larvas de peces con factores ambientales}

Se observó una correlación positiva entre la densidad de huevos de A. mitchilli, C. edentulus y Achiridae con la temperatura y la salinidad, mientras que la densidad de huevos de Perciformes solo correlacionó con la salinidad. En ambas especies de engráulidos la correlación positiva con la temperatura concuerda con la temporada de desove, la cual ocurre principalmente durante la época cálida del año (tablas 2 y 3). Existió correlación positiva entre la densidad de larvas de A. mitchilli y Membras martinica con la temperatura, B. gunteri y L. rhomboides con la salinidad, mientras que la de Lupinoblennius nicholsi y Ctenogobius boleosoma correlacionó con ambos parámetros (tabla 3).

\section{Parámetros ecológicos}

La diversidad de larvas de peces fue mayor durante el mes de mayo, correspondiente a la temporada de secas ( 3.30 bits/ind), y el menor valor ( 2.13 bits/ind) se obtuvo en la época fría -febrero-, cuando la riqueza específica fue mayor (26) por el ingreso de especies a la laguna de Tamiahua por efecto de los «nortes» (tabla 2). La dominancia fue mayor durante el mes de febrero debido principalmente al ingreso de L. rhomboides y B. gunteri, y a la gran abundancia de larvas de A. mitchi$l l i, C$. boleosoma y M. martinica, especies que se presentaron a lo largo del año, con su principal periodo reproductivo en esta época. Por otro lado, la equidad en la comunidad alcanzó el mayor valor (0.78) en noviembre, cuando la dominancia es menor (0.17) y la diversidad fue alta (3.21). De acuerdo con el IVI $\geq 7.48$, las especies dominantes fueron: A. mitchilli, L. rhomboides, M. martinica, L. nicholsi, C. boleosoma y B. gunteri (tabla 2).

\section{Abundancia espacial del ictioplancton en la laguna de Tamiahua}

Al comparar las 2 subregiones del sistema, se observó que los huevos penetran principalmente por la boca de Tampachichi, y el desove de $A$. mitchilli, aunque ocurre en toda la laguna, presenta la mayor densidad en la región norte (tabla 4), a diferencia de lo observado con las larvas de peces que únicamente fueron más abundantes en la región norte durante febrero y mayo,

Tabla 3

Correlación de rangos de Spearman $\left(\mathrm{r}_{\mathrm{s}}\right)$ entre la densidad de huevos más abundantes y larvas de peces dominantes $(\mathrm{IVI}>7.4 \%)$ con la temperatura $\left({ }^{\circ} \mathrm{C}\right)$ y la salinidad superficial de la laguna de Tamiahua, Veracruz, México (2011).

\begin{tabular}{|c|c|c|c|c|}
\hline & $\begin{array}{l}\text { Temperatura }\left({ }^{\circ} \mathrm{C}\right) \\
\mathrm{r}_{\mathrm{s}}\end{array}$ & $\begin{array}{l}\text { Salinidad (ups) } \\
\mathrm{r}_{\mathrm{s}}\end{array}$ & $\mathrm{r}_{\mathrm{s}}$ tablas & $\alpha$ \\
\hline \multicolumn{5}{|l|}{ Huevos } \\
\hline Anchoa mitchilli & $0.580^{\mathrm{a}}$ & $0.346^{\mathrm{a}}$ & 0.210 & 0.05 \\
\hline Cetengraulis edentulus & $0.439^{\mathrm{a}}$ & $0.459^{\mathrm{a}}$ & & \\
\hline Perciformes & 0.166 & $0.724^{\mathrm{a}}$ & & \\
\hline Achiridae & $0.443^{\mathrm{a}}$ & $0.463^{\mathrm{a}}$ & & \\
\hline \multicolumn{5}{|l|}{ Larvas } \\
\hline Anchoa mitchilli & $0.227^{\mathrm{a}}$ & 0.073 & & \\
\hline Brevoortia gunteri & 0.153 & $0.256^{\mathrm{a}}$ & & \\
\hline Membras martinica & $0.308^{\mathrm{a}}$ & 0.181 & & \\
\hline Lagodon rhomboides & 0.167 & $0.504^{\mathrm{a}}$ & & \\
\hline Lupinoblennius nicholsi & $0.385^{\mathrm{a}}$ & $0.489^{\mathrm{a}}$ & & \\
\hline Ctenogobius boleosoma & $0.409^{\mathrm{a}}$ & $0.599^{\mathrm{a}}$ & & \\
\hline
\end{tabular}

$\alpha$ : nivel de significación; IVI: índice de valor de importancia, riqueza específica, diversidad, dominancia y equidad.

${ }^{\text {a }}$ Correlación significativa. 
Tabla 4

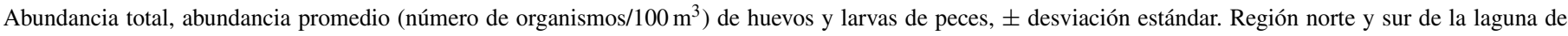
Tamiahua y comparación entre regiones a través del índice de Schoener (\%).

\begin{tabular}{|c|c|c|c|c|c|c|c|}
\hline & \multicolumn{3}{|c|}{ Norte } & \multicolumn{3}{|c|}{ Sur } & \multirow[b]{2}{*}{$\%$} \\
\hline & Total & Promedio & $\mathrm{DE}$ & Total & Promedio & $\mathrm{DE}$ & \\
\hline \multicolumn{8}{|l|}{ Huevos } \\
\hline Febrero & $1,180.6$ & 118.1 & 208.6 & 14.1 & 1.2 & 3.1 & 73.5 \\
\hline Mayo & $20,073.9$ & $2,007.4$ & $3,442.8$ & $5,772.4$ & 481.0 & $1,154.1$ & 83.2 \\
\hline Agosto & $14,627.5$ & $1,462.7$ & $2,975.9$ & $8,078.5$ & 673.2 & $1,624.0$ & 79.3 \\
\hline Noviembre & $3,803.3$ & 380.3 & 876.8 & $1,580.1$ & 131.7 & 418.1 & 70.1 \\
\hline \multicolumn{8}{|l|}{ Larvas } \\
\hline Febrero & $1,024.7$ & 102.5 & 310.3 & 565.7 & 47.1 & 99.8 & 9.6 \\
\hline Mayo & 476.4 & 47.6 & 75.4 & 147.9 & 12.3 & 18.0 & 29.1 \\
\hline Agosto & 121.1 & 12.1 & 16.2 & 416.5 & 34.7 & 45.9 & 24.0 \\
\hline Noviembre & 69.0 & 6.9 & 7.0 & 128.5 & 10.7 & 16.2 & 45.7 \\
\hline
\end{tabular}

DE: desviación estándar.

atribuido principalmente a las especies dominantes: L. rhomboides, B. gunteri y A. mitchilli. Al comparar las regiones norte y sur a través de la densidad de las especies a nivel larvario (tabla 4), el traslapamiento va de bajo a moderado; el valor menor $(9.6 \%)$ ocurrió en febrero, debido a que únicamente 4 especies fueron comunes, 15 exclusivas de la región norte y 7 de la región sur.

\section{Asociación de especies}

De acuerdo con el índice de Bray-Curtis la comunidad de larvas de peces quedó conformada por 7 grupos (fig. 2): Grupo I) integrado por especies exclusivas en el mes de noviembre (Trinectes maculatus y Gobionellus oceanicus) o con su mayor abundancia registrada en ese mes, como Micropogonias undulatus; Grupo II) especies presentes todo el año o en la mayor parte de este, con un valor de IVI de 2.81 a 8.38\%; Grupo III) especies con abundancia relativa baja (0.94 a 1.23\%), principalmente en febrero; Grupo IV) tres especies dominantes: A. mitchilli, L. rhomboides y B. gunteri, con la mayor densidad en febrero, las 2 primeras con los mayores valores de IVI, 38.16 y $37.20 \%$, respectivamente; Grupo V) especies exclusivas en mayo: $C$. edentulus, $C$. shufeldti, una especie de Serranidae, Trachinotus falcatus, Opisthonema oglinum e Hyporhamphus unifasciatus, o con la mayor abundancia en este mes: Anchoa hepsetus, D. maculatus y Evorthodus lyricus; Grupo VI) constituido por especies exclusivas de febrero con densidades muy bajas: Strongylura marina, Archosargus probatocephalus, Eleotris pisonis, M. thalassinus, Diplodus sp., Elops saurus, C. arctifrons y Gobiesox strumosus; Grupo VII) congregó a las especies exclusivas de agosto: Microphis brachyurus lineatus, L. campechanus y Diapterus rhombeus, con densidades muy bajas. Por último, fueron excluidas de los grupos Stellifer lanceolatus y Bathygobius soporator, con valores muy bajos de densidad.

\section{Riqueza específica entre lagunas costeras del noreste de México}

Al comparar la riqueza específica de larvas de peces de la laguna de Tamiahua antes y después de que la boca de
Tampachichi fuera abierta de forma permanente (tabla 5) se observó una similitud del $61 \%$, menor a lo obtenido con las lagunas Madre y Tampamachoco (76 y 70\%, respectivamente).

\section{Discusión}

De acuerdo con el Sistema de Venecia (1958), la laguna de Tamiahua presentó condiciones polieuhalinas, a diferencia de lo señalado por Flores-Coto et al. (1983), quienes la consideraban polihalina. En mayo se registraron los máximos valores, de 34.0 a 37.0 ups en ambas bocas de comunicación con el mar y su zona de influencia, mientras que la salinidad más baja (2.0 ups) se registró en agosto frente a Tampico Alto, al norte de la laguna.

El desove de $A$. mitchilli ocurrió durante todo el ciclo anual con un pico en verano-otoño y la menor densidad en febrero; se observa que la densidad del desove es considerablemente menor (45\% del total de huevos) con respecto a lo obtenido por Flores-Coto et al. (1983), quienes obtuvieron un $72 \%$ con un pico en el invierno, mientras que en las lagunas de Tampamachoco y Pueblo Viejo el desove fue mayor, con un 84 y un 98\%, respectivamente (Ocaña-Luna y Sánchez-Ramírez, 2003; Sánchez-Ramírez y Ocaña-Luna, 2002). La disminución en la densidad de huevos en la laguna de Tamiahua durante febrero probablemente se debió a las bajas temperaturas por efecto de los «nortes» en esa época del año, con un intervalo de $13-18.5^{\circ} \mathrm{C}$ (media de $15 \pm 1.9^{\circ} \mathrm{C}$ ), además de presentar una correlación positiva entre la densidad de huevos con la temperatura, lo que corresponde con lo observado por Monteleone (1992), quien señala que en regiones de mayor latitud el periodo de desove se desfasa de mayo a agosto en temperaturas y salinidades $>15^{\circ} \mathrm{C}$ y 20 ups, respectivamente.

Cetengraulis edentulus ha sido registrada con su límite de distribución al norte hasta la laguna Pueblo Viejo (CastilloRivera, Ortiz-Burgos y Zárate-Hernández, 2011). En la laguna de Tamiahua la mayor abundancia de huevos se presentó durante la época cálida, con una densidad anual relativa del $7.57 \%$, mayor a lo registrado con anterioridad $(0.17 \%)$ en la misma laguna por Flores-Coto et al. (1983) y en la laguna de Tampamachoco $(0.01 \%)$ señalado por Ocaña-Luna y Sánchez-Ramírez (2003). En la laguna de Términos, Campeche, desova todo el 
Tabla 5

Especies de larvas de peces recolectadas en lagunas costeras de Tamaulipas y Veracruz, México.

\begin{tabular}{|c|c|c|c|c|}
\hline Especies & LAMA & TAMI & TAMI (1) & TAMPA \\
\hline Elops saurus Linnaeus, 1766 & $\mathrm{X}$ & $\mathrm{X}$ & $\mathrm{X}$ & $\mathrm{X}$ \\
\hline Myrophis punctatus Lütken, 1852 & $\mathrm{X}$ & & $\mathrm{X}$ & $\mathrm{X}$ \\
\hline Anchoa hepsetus (Linnaeus, 1758) & $\mathrm{X}$ & $\mathrm{X}$ & $\mathrm{X}$ & $\mathrm{X}$ \\
\hline Anchoa mitchilli (Valenciennes, 1848) & $\mathrm{X}$ & $\mathrm{X}$ & $\mathrm{X}$ & $\mathrm{X}$ \\
\hline Cetengraulis edentulus (Cuvier, 1829) & & $\mathrm{X}$ & & \\
\hline Brevoortia gunteri Hildebrand, 1948 & $X$ & $\mathrm{X}$ & & \\
\hline Harengula jaguana Poey, 1865 & $\mathrm{X}$ & & $\mathrm{X}$ & $\mathrm{X}$ \\
\hline Opisthonema oglinum (Lesueur, 1818) & $\mathrm{X}$ & $\mathrm{X}$ & & \\
\hline Synodus foetens (Linnaeus, 1766) & $\mathrm{X}$ & & & \\
\hline Membras martinica (Valenciennes, 1835) & $\mathrm{X}$ & $X$ & $\mathrm{X}$ & $\mathrm{X}$ \\
\hline Menidia beryllina (Cope, 1867$)$ & $\mathrm{X}$ & & & \\
\hline Hyporhamphus unifasciatus (Ranzani, 1841) & $\mathrm{X}$ & $\mathrm{X}$ & $\mathrm{X}$ & \\
\hline Strongylura marina (Walbaum, 1792) & $\mathrm{X}$ & $\mathrm{X}$ & $\mathrm{X}$ & \\
\hline Strongylura notata (Poey, 1860) & & & $\mathrm{X}$ & \\
\hline Hippocampus zosterae Jordan y Gilbert, 1882 & $\mathrm{X}$ & & & \\
\hline Microphis brachyurus lineatus (Kaup, 1856) & & $\mathrm{X}$ & $\mathrm{X}$ & \\
\hline Syngnathus floridae (Jordan y Gilbert, 1882) & $\mathrm{X}$ & & & \\
\hline Syngnathus louisianae Günther, 1870 & & $X$ & $X$ & \\
\hline Syngnathus scovelli (Evermann y Kendall, 1896) & $\mathrm{X}$ & $\mathrm{X}$ & $\mathrm{X}$ & $\mathrm{X}$ \\
\hline Oligoplites saurus (Bloch y Schneider, 1801) & $\mathrm{X}$ & $\mathrm{X}$ & $\mathrm{X}$ & \\
\hline Trachinotus falcatus (Linnaeus, 1758) & & $\mathrm{X}$ & & \\
\hline Lutjanus campechanus (Poey, 1860) & & $\mathrm{X}$ & & \\
\hline Diapterus auratus Ranzani, 1842 & & & $\mathrm{X}$ & \\
\hline Diapterus rhombeus (Cuvier, 1829) & $\mathrm{X}$ & $\mathrm{X}$ & & $\mathrm{X}$ \\
\hline Eucinostomus melanopterus (Bleeker, 1863) & & & $\mathrm{X}$ & \\
\hline Ulaema lefroyi (Goode, 1874$)$ & $\mathrm{X}$ & $\mathrm{X}$ & & $\mathrm{X}$ \\
\hline Archosargus probatocephalus (Walbaum, 1792) & & $\mathrm{X}$ & $\mathrm{X}$ & \\
\hline Lagodon rhomboides (Linnaeus, 1766) & $\mathrm{X}$ & $\mathrm{X}$ & $\mathrm{X}$ & $\mathrm{X}$ \\
\hline Polydactylus octonemus (Girard, 1858) & & & & $\mathrm{X}$ \\
\hline Bairdiella chrysoura (Lacépède, 1802) & $\mathrm{X}$ & $\mathrm{X}$ & $\mathrm{X}$ & $\mathrm{X}$ \\
\hline Cynoscion arenarius Ginsburg, 1930 & $\mathrm{X}$ & $\mathrm{X}$ & $\mathrm{X}$ & $\mathrm{X}$ \\
\hline Cynoscion nebulosus (Cuvier, 1830) & $\mathrm{X}$ & $\mathrm{X}$ & $\mathrm{X}$ & $\mathrm{X}$ \\
\hline Leiostomus xanthurus Lacépède, 1802 & $\mathrm{X}$ & $\mathrm{X}$ & $\mathrm{X}$ & $\mathrm{X}$ \\
\hline Menticirrhus littoralis (Holbrook, 1847) & $\mathrm{X}$ & & & \\
\hline Micropogonias undulatus (Linnaeus, 1766) & & $\mathrm{X}$ & & $\mathrm{X}$ \\
\hline Stellifer lanceolatus (Holbrook, 1855) & $\mathrm{X}$ & $\mathrm{X}$ & & \\
\hline Chasmodes bosquianus (Lacépède, 1800) & & $\mathrm{X}$ & & \\
\hline Lupinoblennius nicholsi (Tavolga, 1954) & $\mathrm{X}$ & $\mathrm{X}$ & $\mathrm{X}$ & $\mathrm{X}$ \\
\hline Gobiesox strumosus Cope, 1870 & $\mathrm{X}$ & $\mathrm{X}$ & $\mathrm{X}$ & \\
\hline Dormitator maculatus (Bloch, 1792) & $\mathrm{X}$ & $\mathrm{X}$ & $\mathrm{X}$ & $\mathrm{X}$ \\
\hline Eleotris pisonis (Gmelin, 1789) & & $\mathrm{X}$ & & $\mathrm{X}$ \\
\hline Bathygobius soporator (Valenciennes, 1837) & $\mathrm{X}$ & $\mathrm{X}$ & & $\mathrm{X}$ \\
\hline Ctenogobius boleosoma (Jordan y Gilbert, 1882) & $\mathrm{X}$ & $\mathrm{X}$ & & $\mathrm{X}$ \\
\hline Ctenogobius shufeldti (Jordan y Eigenmann, 1887) & $\mathrm{X}$ & $\mathrm{X}$ & & \\
\hline Evorthodus lyricus (Girard, 1858) & $\mathrm{X}$ & $\mathrm{X}$ & & $\mathrm{X}$ \\
\hline Gobioides broussonnetii Lacépède, 1800 & & & $\mathrm{X}$ & $\mathrm{X}$ \\
\hline Gobionellus oceanicus (Pallas, 1770) & $\mathrm{X}$ & $\mathrm{X}$ & & $\mathrm{X}$ \\
\hline Gobiosoma bosc (Lacépède, 1800) & $\mathrm{X}$ & $\mathrm{X}$ & $\mathrm{X}$ & $\mathrm{X}$ \\
\hline Gobiosoma robustum Ginsburg, 1933 & $\mathrm{X}$ & & $\mathrm{X}$ & \\
\hline Microgobius gulosus (Girard, 1858) & & & $\mathrm{X}$ & \\
\hline Microgobius thalassinus (Jordan y Gilbert, 1883) & $\mathrm{X}$ & $\mathrm{X}$ & & \\
\hline Citharichthys arctifrons Goode, 1880 & $\mathrm{X}$ & $\mathrm{X}$ & & $\mathrm{X}$ \\
\hline Citharichthys spilopterus Günther, 1862 & & & $\mathrm{X}$ & $\mathrm{X}$ \\
\hline Achirus lineatus (Linnaeus, 1758) & $\mathrm{X}$ & $\mathrm{X}$ & $\mathrm{X}$ & $\mathrm{X}$ \\
\hline Trinectes maculatus (Bloch y Schneider, 1801) & & $\mathrm{X}$ & $\mathrm{X}$ & $\mathrm{X}$ \\
\hline Acanthostracion quadricornis (Linnaeus, 1758) & $\mathrm{X}$ & & & \\
\hline Sphoeroides parvus Shipp y Yerger, 1969 & & & $\mathrm{X}$ & \\
\hline
\end{tabular}

LAMA = laguna Madre, Tamaulipas (Sánchez-Ramírez y Ocaña-Luna, 2015); TAMI = laguna de Tamiahua, Veracruz (presente estudio); TAMI (1)=laguna de Tamiahua, Veracruz (Sanvicente-Añorve et al., 2011); TAMPA = laguna de Tampamachoco, Veracruz (Ocaña-Luna y Sánchez-Ramírez, 2003). 
año con su mayor abundancia en los meses de enero a septiembre con un pico en febrero $\left(1,095.71\right.$ huevos $\left./ 100 \mathrm{~m}^{3}\right)$ y con una abundancia de huevos similar a A. mitchilli (49.82\%) (FloresCoto, Ocaña-Luna, Luna-Calvo y Zavala-García, 1988). En el hemisferio sur el periodo reproductivo de esta especie de afinidad tropical ocurre principalmente en primavera-verano (Silva, Araujo, de Azevedo y Mendonça, 2003; Souza-Conceiçao, Rodrigues-Ribeiro y Castro-Silva, 2005), mientras que en el Caribe colombiano sucede en 2 épocas: la primera, de abril a junio, y la segunda, de noviembre a diciembre (Osorio-Dualiby y Báez-Hidalgo, 2002).

Lutjanus campechanus ha sido registrado en la laguna Madre, Tamaulipas, por Hildebrand (1958), y en la desembocadura del Río Grande por Edwards y Contreras-Balderas (1991); es común en el norte del golfo de México hasta la costa norte de la península de Yucatán (Castro-Aguirre, Espinosa-Pérez y Schmitter-Soto, 1999).

El primer registro de C. bosquianus en México fue realizado por Castillo-Rivera, Zárate-Hernández y Ortiz-Burgos (2005) en la laguna de Pueblo Viejo. Se distribuye en el océano Atlántico occidental en los estados de Virginia, Carolina del Norte y Carolina del Sur, así como en el norte del golfo de México. En el estado de Luisiana, Estados Unidos de América, es considerada como una especie marina en tránsito con algunas poblaciones residentes permanentes en estuarios (Nordlie, 2003). En la laguna Madre de Texas es una especie común, particularmente en pastos marinos, bancos de ostiones y raíces de manglar
(McKee, 2008). Las larvas de C. shufeldti, M. thalassinus y C. arctifrons fueron registradas previamente en la laguna Madre por Sánchez-Ramírez y Ocaña-Luna (2015).

Las especies dominantes (IVI $\geq 7.79 \%$ ) A. mitchilli, M. martinica, L. nicholsi y C. boleosoma han sido consideradas por Sanvicente-Añorve et al. (2011) residentes permanentes en sistemas estuarinos lagunares del sur del golfo de México y Caribe mexicano, debido a que realizan todo su ciclo de vida en estos ambientes, lo que coincide con el presente estudio; presentaron correlación positiva con la temperatura, debido a que la mayor densidad larvaria se distribuye en aguas cálidas; por otro lado, B. gunteri y L. rhomboides se correlacionaron con la salinidad, ya que sus larvas se registraron principalmente en las bocas y su zona de influencia marina; son especies que usualmente viven en el mar, pero sus larvas y/o juveniles hacen uso extensivo de la laguna de Tamiahua, consideradas como estuarinodependientes por Castillo-Rivera y Kobelkowsky (2000) y Reese, Stunz y Bushon (2008). En particular L. rhomboides está considerada como dominante en etapa larvaria que migra a los estuarios y/o lagunas costeras durante otoño-invierno para completar su desarrollo en el océano Atlántico occidental y norte del golfo de México (Forward, Jr., Tankersley y Reinsel, 1998; Reese et al., 2008; Warlen y Burke, 1990).

En la laguna de Tamiahua no se registraron especies dulceacuícolas, aunque las larvas de A. mitchilli, D. maculatus y $G$. bosc fueron recolectadas con muy baja abundancia en agosto en condiciones oligohalinas al norte de la laguna, donde existe

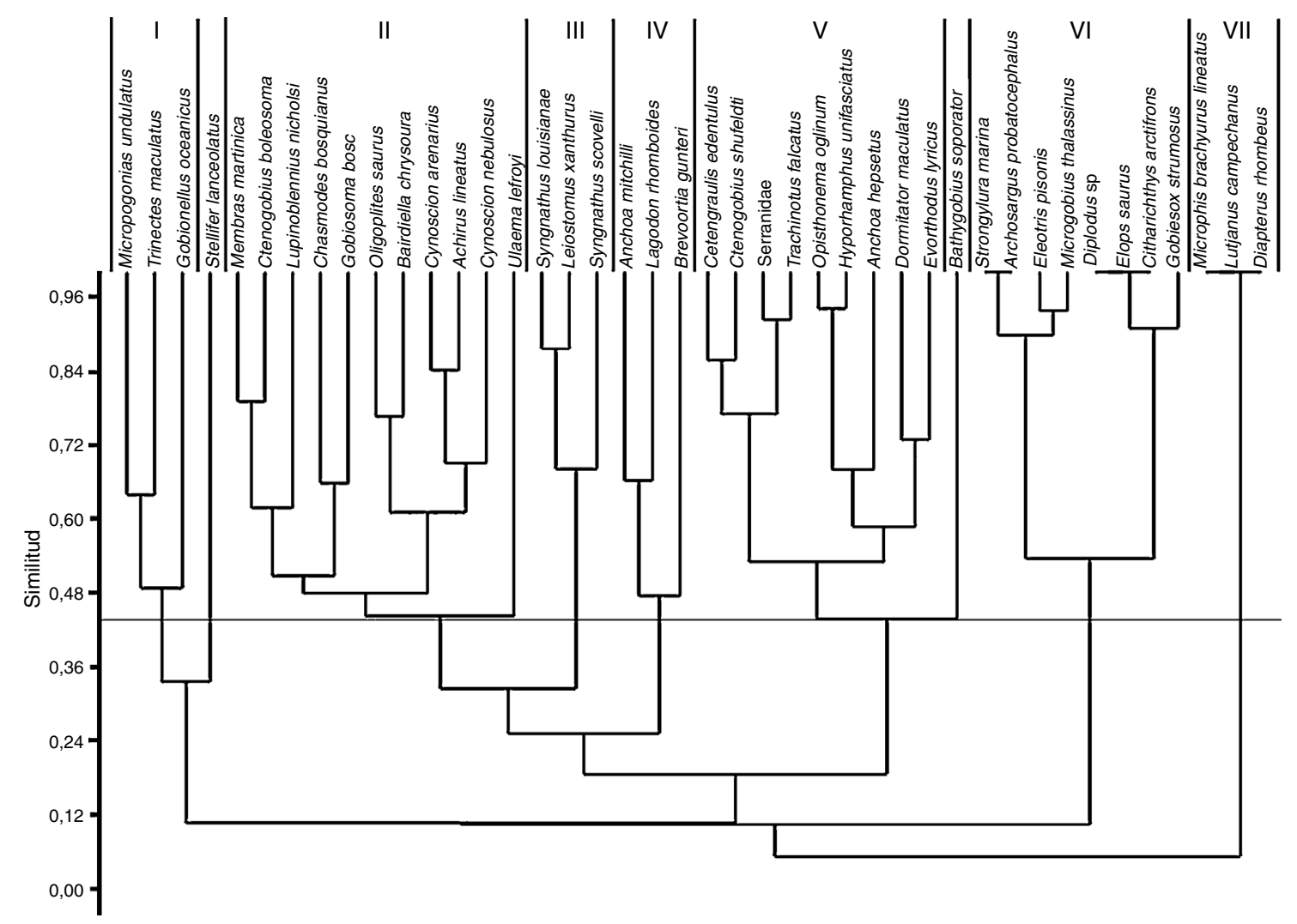

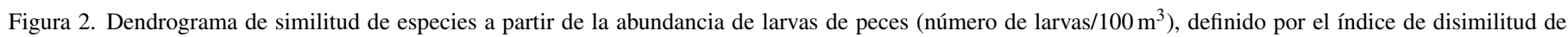
Bray-Curtis, grupos pareados. Laguna de Tamiahua, Veracruz, México. 
la influencia del río Pánuco; Miller, Minckley y Norris (2005) mencionan que pueden penetrar a los ríos junto con $B$. gunteri, M. martinica, S. marina, M. brachyurus lineatus, D. rhombeus, M. undulatus, E. lyricus y T. maculatus. En esta laguna, cuando la boca de Tampachichi era intermitente y con condiciones polihalinas, se registraron de 20 a 32 especies de peces de acuerdo con Flores-Coto et al. (1983) y Sanvicente-Añorve et al. (2011), a diferencia de las 40 especies determinadas actualmente, cuando la boca de la laguna está abierta permanentemente, lo que permite un mayor ingreso de especies marinas. Se observa una mayor similitud con la laguna Madre (39), laguna hipersalina con un fuerte componente de especies marinas señaladas por Sánchez-Ramírez y Ocaña-Luna (2015). Por otro lado, la mayor riqueza en la laguna de Tamiahua es menor a la obtenida en un estuario subtropical de las costas de Florida, donde Reyier y Shenker (2007) señalaron 58 especies, y en la laguna Madre de Texas, con 55 (Tolan, Holt y Onuf, 1997), lo que puede deberse principalmente a la gran heterogeneidad de hábitats que presentan.

La mayor diversidad de larvas de peces en la laguna de Tamiahua fue en primavera y otoño, a diferencia de lo observado por Flores-Coto et al. (1983), quienes registran las mayores en otoño e invierno; en la laguna de Tampamachoco la diversidad fue similar en primavera-otoño (Ocaña-Luna y Sánchez-Ramírez, 2003); mientras que en la laguna Madre ocurrió en diferentes épocas -invierno y verano- (Sánchez-Ramírez y Ocaña-Luna, 2015), en general en las lagunas costeras del noreste de México, la disminución de la diversidad del ictioplancton en algunas épocas del año es debida principalmente a la gran abundancia de las especies dominantes: A. mitchilli y/o A. hepsetus.

El cierre de barras altera la salinidad y favorece las condiciones oligohalinas con el incremento de la abundancia de macrófitas acuáticas, promoviendo la dominancia de especies de peces dulceacuícolas y la reducción de especies marinodependientes y de algunas estuarinodependientes adaptadas a ecosistemas costeros (Sánchez-Botero, Pellegrini-Caramaschi y Sequeira-Garcez, 2008); en particular Strydom et al. (2003), con estudios de larvas y juveniles de peces en estuarios con bocas abiertas intermitentemente, los caracterizaron con baja riqueza, diversidad y dominancia de especies estuarinorresidentes, mientras que los sistemas con bocas abiertas permanentemente tuvieron predominancia de larvas y juveniles de especies marinas estuarinodependientes. En la laguna de Tamiahua, con ambas bocas permanentemente abiertas, se observa un incremento en la salinidad (polieuhalinas) que favorece el ingreso de especies marinas estuarinodependientes, con la mayor riqueza específica en febrero (26), especies exclusivas (10), especies dominantes (L. rhomboides, A. mitchilli y B. gunteri) y con la mayor abundancia larvaria en la región norte de la laguna en febrero, lo que puede atribuirse a la sincronización de los periodos reproductivos de las especies de peces marinos en otoño-invierno, que inmigran a los estuarios y lagunas costeras como lo han mencionado Hoss, Coston-Clements, Peters y Tester (1988); Reese et al. (2008) y Warlen y Burke (1990); esta inmigración puede ser favorecida por la circulación oceánica desde Luisiana y Texas a Tamaulipas y Veracruz, de septiembre a marzo, que señalan Zavala-Hidalgo, Morey y O'Brien (2003).

\section{Agradecimientos}

Al Instituto Politécnico Nacional por las becas otorgadas a los autores a través de los programas: Estímulo al Desempeño de los Investigadores (EDI) y Comisión de Operación y Fomento a las Actividades Académicas (COFAA), así como al Sistema Nacional de Investigadores del Conacyt, a la Secretaría de Investigación y Posgrado (SIP) del IPN por el financiamiento a los proyectos: SIP-20110263 y SIP-20144447, para realizar esta investigación. A Sonia Sánchez-Serano por la determinación de larvas de la familia Engraulidae y al personal de la Sociedad Cooperativa de Producción Pesquera La Rivera de Tampico Alto por su gran apoyo en el trabajo de campo.

\section{Referencias}

Abarca-Arenas, L. G., Franco-López, J., González-Gándara, C. y Silva-López, G. (2012). Los peces de la costa veracruzana: relación especies-área y conectividad entre sitios. En L. F. del Moral-Flores, J. A. Martínez-Pérez, J. Franco-López, Á. J. Ramírez-Villalobos y J. L. Tello-Musi (Eds.), Investigación ictiológica en México. Temas selectos en honor al Dr. José Luis Castro Aguirre (pp. 127-158). México, D. F.: Universidad Nacional Autónoma de México, Facultad de Estudios Superiores Iztacala y Sociedad Ictiológica Mexicana, A. C.

Ayala-Castañares, A., Cruz, R., García-Cubas, A., Jr. y Segura, L. R. (1969). pp. 39-48. Síntesis de conocimientos sobre la geología marina de la laguna de Tamiahua, Veracruz, México. Lagunas costeras, un simposio: memorias del Simposio Internacional de Lagunas Costeras. Nov. 28-30, 1967 México, D. F.: UNAM-UNESCO.

Ayala-Castanares, A. y Phleger, F. B. (Eds.). (1969). Lagunas costeras, un simposio: memorias del Simposio Internacional de Lagunas Costeras (origen, dinámica y productividad). Nov. 28-30, 1967. México, D. F.: UNAMUNESCO.

Bray, J. R. y Curtis, J. T. (1957). An ordination of the upland forest communities of Southern Wisconsin. Ecological Monographs, 27, 325-349.

Briggs, J. C. (1995). Global biogeography. Developments in paleontology and stratigraphy. Amsterdam: Elsevier.

Castillo-Rivera, M. y Kobelkowsky, A. (2000). Distribution and segregation of two sympatric Brevoortia species (Teleostei: Clupeidae). Estuarine, Coastal and Shelf Science, 50, 593-598.

Castillo-Rivera, M., Ortiz-Burgos, S. y Zárate-Hernández, R. (2011). Estructura de la comunidad de peces estuarinos en un hábitat con vegetación sumergida: variación estacional nictímera. Hidrobiológica, 21, 311-321.

Castillo-Rivera, M., Zárate-Hernández, R. y Ortiz-Burgos, S. (2005). Variación nictímera y estacional de la abundancia, riqueza y especies dominantes de peces, en un ambiente salobre de sustrato blando. Hidrobiológica, 15, 227-238.

Castro-Aguirre, J. L., Espinosa-Pérez, H. S. y Schmitter-Soto, J. J. (1999). Ictiofauna estuarino-lagunar y vicaria de México. México, D. F.: Editorial Limusa.

Contreras-Espinosa, F. (1993). Ecosistemas costeros mexicanos. México, D. F.: Comisión Nacional para el Conocimiento y Uso de la Biodiversidad y Universidad Autónoma Metropolitana, Unidad Iztapalapa.

Day, J. y Yáñez-Arancibia, A. (1982). Coastal lagoons and estuaries: ecosystem approach. Ciencia Interamericana, 22, 11-26.

Díaz-Ávalos, C., Barba-Torres, J. F. y Gaspar-Dillanes, M. T. (2003). Variables ambientales y abundancia de los huevos de Anchoa mitchilli (Pisces: Engraulidae) en la laguna de Tamiahua, México. Revista Biología Tropical, 51, 471-478.

Edwards, R. J. y Contreras-Balderas, S. (1991). Historical changes in the ichthyofauna of the lower Rio Grande (Río Bravo del Norte), Texas and Mexico. Southwestern Naturalist, 36, 201-212.

Flores-Coto, C., Barba-Torres, F. y Sánchez-Robles, J. (1983). Seasonal diversity, abundance, and distribution of ichthyoplankton in Tamiahua Lagoon, 
Western Gulf of Mexico. Transactions of the American Fisheries Society, $112,247-256$.

Flores-Coto, C., Ocaña-Luna, A., Luna-Calvo, A. y Zavala-García, F. (1988). Abundancia de algunas especies de anchoas en la laguna de Términos (México), estimada a través de la captura de huevos. Anales del Instituto de Ciencias del Mar y Limnología, Universidad Nacional Autónoma de México, 15, 125-134.

Forward, R. B., Jr., Tankersley, R. A. y Reinsel, K. A. (1998). Selective tidal stream transport of spot (Leiostomus xanthurus Lacepède) and pinfish [Lagodon rhomboides (Linnaeus)] larvae: contribution of circatidal rhythms in activity. Journal of Experimental Marine Biology and Ecology, 226, $19-32$.

Gaspar-Dillanes, M. T., Sánchez-Iturbe, A., González-Yoval, P. y López-García, D. (1995). Dinámica poblacional y biomasa desovante de la anchoa (Anchoa mitchilli) en la laguna de Tamiahua, Veracruz, México, en 1985 y 1986. Ciencia Pesquera, 11, 21-27.

Gómez-Aguirre, S. y Reséndez-Medina, A. (1986). Notas sobre la hidrobiología del sistema de lagunas costeras Carmen-Machona-Redonda, Tabasco, 19761980. Universidad y Ciencia, 3, 5-10.

Hammer, Ø., Harper, D. A. T. y Ryan, P. D. (2001). PAST: Paleontological statistics software package for education and data analysis. Palaeontologia Electronica, 4. Disponible en: http://folk.uio.no/ohammer/past

Hildebrand, H. H. (1958). Estudios biológicos preliminares sobre la laguna Madre de Tamaulipas. Ciencia, 17, 151-173.

Hoss, D. E., Coston-Clements, L., Peters, D. S. y Tester, P. A. (1988). Metabolic responses of spot Leiostomus xanthurus, and Atlantic croaker, Micropogonias undulatus, larvae to cold temperatures encountered following recruitment to estuaries. Fishery Bulletin, 86, 483-488.

Kjerfve, B. (1994). Coastal lagoons. En B. Kjerfve (Ed.), Coastal lagoons processes (60) (pp. 1-8). Amsterdam: Elsevier Oceanography Series.

Kjerfve, B. y Magill, K. E. (1989). Geographic and hydrodynamic characteristics of shallow coastal lagoons. Marine Geology, 88, 187-199.

Krebs, C. (1985). Ecología: estudio de la distribución y la abundancia (segunda edición). México, D. F.: Harla.

Lankford, R. R. (1977). Coastal lagoons for Mexico. Their origin and classification. En M. Wiley (Ed.), Estuarine processes (pp. 182-218). New York: Academic Press Incorporation.

McKee, D. A. (2008). Fishes of the Texas Laguna Madre. Hong Kong: Everbest Printing Co.

Miller, R. R., Minckley, W. L. y Norris, S. M. (2005). Freshwater fishes of Mexico. Chicago: University of Chicago Press.

Monteleone, D. M. (1992). Seasonality and abundance of ichthyoplankton in Great South Bay, New York. Estuaries, 15, 230-238.

Nordlie, F. G. (2003). Fish communities of estuarine salt marshes of eastern North America, and comparisons with temperate estuaries of other continents. Reviews in Fish Biology and Fisheries, 13, 281-325.

Ocaña-Luna, A. y Sánchez-Ramírez, M. (1998). Feeding of Sciaenid (Pisces: Sciaenidae) larvae in two coastal lagoons of the Gulf of Mexico. Gulf Research Report, 10, 1-9.

Ocaña-Luna, A. y Sánchez-Ramírez, M. (2003). Diversity of ichthyoplankton in Tampamachoco Lagoon, Veracruz, Mexico. Anales del Instituto de BiologíaUniversidad Nacional Autónoma de México. Serie Zoología, 74, 179-193.

Osorio-Dualiby, D. y Báez-Hidalgo, M. (2002). Reproducción de la bocona Cetengraulis edentulus (Cuvier, 1829) (Pisces: Clupeiformes) en el sector costero isla de Salamanca, Caribe Colombiano. Investigaciones Marinas, 23, 129-139.

Paperno, R., Mille, K. J. y Kadison, E. (2001). Patterns in species composition of fish and selected invertebrate assemblages in estuarine subregions near Ponce de Leon Inlet, Florida. Estuarine, Coastal and Shelf Science, 52, $117-130$.

Pielou, E. C. (1975). Ecological diversity. New York: John Wiley \& Sons.

Reese, M. M., Stunz, G. W. y Bushon, A. M. (2008). Recruitment of estuarinedependent nekton through a new tidal inlet: the opening of Packery Channel in Corpus Christi, TX, USA. Estuaries and Coast, 31, 1143-1157.

Reyier, E. A. y Shenker, J. M. (2007). Ichthyoplankton community structure in a shallow subtropical estuary of the Florida Atlantic Coast. Bulletin of Marine Science, 80, 267-293.
Rodríguez-de la Cruz, M. C. y Palacio-Fest, M. R. (1996). Alteraciones en la productividad de Tamiahua, Veracruz, por cambios hidrodinámicos y desarrollo industrial. Oceanología, 1, 145-153.

Sagarpa-Conapesca (Secretaría de Agricultura, Ganadería, Desarrollo Rural, Pesca y Alimentación-Comisión Nacional de la Pesca). (2007). Manifestación de impacto ambiental, modalidad particular para la rehabilitación y prolongación de escolleras y dragado en la boca de Corazones, laguna de Tamiahua, municipio de Tamiahua, Veracruz. México, D. F.: SagarpaConapesca.

Sagarpa-Conapesca (Secretaría de Agricultura, Ganadería, Desarrollo Rural, Pesca y Alimentación-Comisión Nacional de la Pesca). (2008). Manifestación de impacto ambiental, modalidad particular para la rehabilitación y prolongación de escolleras y dragado de canales en la boca de Tampachiche, laguna de Tamiahua, Veracruz. México, D. F.: Sagarpa-Conapesca.

Salvadores-Baledón, M. L. y Reséndez-Medina, A. (1990). Modificaciones en la composición ictiofaunística del sistema lagunar El Carmen-Machona Tabasco, por la apertura de boca de Panteones. Universidad y Ciencia, 7 , $5-13$.

Sánchez-Botero, J. I., Pellegrini-Caramaschi, E. y Sequeira-Garcez, D. (2008). Spatiotemporal variation in fish assemblage in a Coastal Lagoon without direct contact with the sea (Southeastern Brazil). Journal of Coastal Research, 24, 225-238.

Sánchez-Ramírez, M. y Ocaña-Luna, A. (2002). Temporal variability in the abundance of the bay anchovy Anchoa mitchilli (Valenciennes, 1848) eggs and spawning biomass in Pueblo Viejo Lagoon, Veracruz, Mexico. Hidrobiológica, 12, 157-162.

Sánchez-Ramírez, M. y Ocaña-Luna, A. (2015). Estructura y variación estacional de la comunidad ictioplanctónica en una laguna hipersalina del oeste del golfo de México: laguna Madre, Tamaulipas. Hidrobiológica, 25, 175-186.

Sanvicente-Añorve, L., Sánchez-Ramírez, M., Ocaña-Luna, A., Flores-Coto, C. y Ordóñez-López, U. (2011). Metacommunity structure of estuarine fish larvae: the role of regional and local processes. Journal of Plankton Research, 33, 179-194.

Schoener, T. W. (1970). Nonsynchronous spatial overlap of lizards in patchy habitats. Ecology, 51, 408-418.

Shannon, C. E. y Weaver, W. (1963). The mathematical theory of communication. Chicago, Urbana: The University of Illinois Press.

Silva, M., Araujo, F. G., de Azevedo, M. C. C. y Mendonça, P. (2003). Distribuiçao espacial e temporal de Cetengraulis edentulus (Cuvier) (Actinopterygii, Engraulidae) na Baía de Sepetiba, Rio de Janeiro, Brasil. Revista Brasileira de Zoologia, 20, 577-581.

Simpson, E. E. (1949). Measurement of diversity. Nature, 163, 688.

Sistema de Venecia. (1958). Symposium on the classification of brackish waters, Venice April 8-14, 1958. Archives Oceanography and Limnology, 11, 1-248.

Sklar, F. H. y Browder, J. A. (1998). Coastal environmental impacts brought about by alterations to freshwater flow in the Gulf of Mexico. Environmental Management, 22, 547-562.

Sorensen, T. (1948). A method of establishing groups of equal amplitude in plant sociology based on similarity of species content and its application to analyses of the vegetation on Danish commons. Biologiske Skrifter, 5, 1-34.

Souza-Conceiçao, J. M., Rodrigues-Ribeiro, M. y Castro-Silva, M. A. (2005). Dinamica populacional, biologia reproductiva e o ictioplancton de Cetengraulis edentulus Cuvier (Pisces, Clupeiformes, Engraulidae) na enseada do Saco dos Limoes, Florianópolis, Santa Catarina, Brasil. Revista Brasileira de Zoologia, 22, 953-961.

Strydom, N. A., Whitfield, A. K. y Wooldridge, T. H. (2003). The role of estuarine type in characterizing early stage fish assemblages in warm temperate estuaries, South Africa. African Zoology, 38, 29-43.

Tolan, J. M., Holt, S. A. y Onuf, C. P. (1997). Distribution and community of ichthyoplankton in Laguna Madre seagrass meadows: potential impact of seagrass species change. Estuaries, 20, 450-464.

Warlen, S. M. y Burke, J. S. (1990). Immigration of larvae of fall/winter spawning marine fishes into a North Carolina Estuary. Estuaries, 13, 452-461.

Zar, J. H. (2010). Biostatistical analysis. New Jersey: Prentice Hall.

Zavala-Hidalgo, J., Morey, S. L. y O'Brien, J. J. (2003). Seasonal circulation on the western shelf of the Gulf of Mexico using a high resolution numerical model. Journal of Geophysical Research, 108, 1-19. 